\title{
Study on the Coupling Mechanism of Urban Spatial form in Coastal City and Mesoscale Wind Environment Based on Multi-variable Analysis-Study Significance, Content, Targets and Innovation Points
}

\author{
$\mathrm{Ke} \mathrm{He}^{1, \mathrm{a}}$, Xiaowan Han ${ }^{2, \mathrm{~b}}$, Yanting $\mathrm{He}^{3, \mathrm{c}}$ and Chunxi Zhao ${ }^{4, \mathrm{~d}}$ \\ ${ }^{1}$ North Gate Construction Building, No. 3688, Nanhai Avenue, Nanshan District, Shenzhen, \\ Guangdong Province \\ ${ }^{2}$ Huaqiao University, No.668, Jimei Avenue, Jimei District, Xiamen, Fujian Province \\ ${ }^{3}$ School of Civil Engineering, Beifang University of Nationalities, No. 204, Wenchang North Street, \\ Xixia District, Yinchuan, Ningxia Hui Autonomous Region \\ ${ }^{4}$ North China University of Technology, No. 5, Jinyuanzhuang Road, Shijingshan District, Beijing \\ ahkqq5@qq.com, b2512034217@qq.com, c15594735@qq.com, d43062282@qq.com
}

\begin{abstract}
Keywords: Mesoscale Wind Environment, Urban Spatial Form, Multi-variable Analysis, Wind Tunnel Experiment, Coupling Mechanism
\end{abstract}

Abstract. For contradiction and complexity of wind environment variables of coastal cities and difficulties in the quantitative research of urban spatial form, the multi-variable analysis technology is flexibly used to explore the feasible technical approach and coupling mechanism for the integration of coastal urban spatial form and the mesoscale wind environment system. The mechanism of influence of changes of coastal urban spatial form on wind environment is summarized, and the energy balance mode based on mesoscale wind environment nested with the urban boundary layer combining with the underlying surface of cities is studied, so as to make strategies for the response by urban spatial form to urban wind environment, simulate and analyze the response characteristics of urban heat island, local wind and pollution diffusion under different scenarios, and determine the feasible scheme of urban spatial form combined with demands for the development of social economy. With the combination of satellite remote sensing and ground monitoring, related parameters are collected, and simulated and calculated with CFD. After a conclusion is drawn through the multi-variable analysis the large wind tunnel laboratory model is used for verification. Further R\&D can optimize the spatial form guidance system software for wind environment of coastal cities. This study will help to actively adjust urban wind environment through space guidance; provide reference for air pollution prevention and control, typhoon prevention and mitigation, urban energy saving and emission reduction and other hot research issues caused by the high-speed development of coastal cities in China; and respond to the government's call for the construction of new smart cities.

\section{Introduction}

The coastal area of China is one of the areas with the most typhoons and disasters in the world, and an area with high urbanization level. In the past 40 years, with the rapid development of cities, the rapid expansion of the scale, the rapid increase of population and denser and denser buildings, urban spatial form undergoes great changes, resulting in urban heat island effect, increased haze, difficult prevention and treatment of typhoon and other wind environment problems ${ }^{1}$. Especially all kinds of buildings erected have changed the urban air channels, intensified the funnelling, caused strong winds around buildings, and deteriorated wind environment around buildings; and coastal cities in China have not developed enough solutions in response to the typhoon, and most of them can only passively defend. If we consider shelter, screen, air channels and other spatial form plans,

\footnotetext{
${ }^{1}$ In 2014 the number of days with haze weather above slightly polluted throughout the year in Dalian is 83 , accounting for $22.7 \%$ of the year; in 2016 the average concentration of PM2.5 in Tianjin was 69 micrograms per cubic meter, exceeding the standard for 0.97 time; the average concentration of PM10 was 103 micrograms per/cubic meter, exceeding the standard for 0.47 time; the average concentration of nitrogen dioxide was 48 micrograms per cubic meter, exceeding the standard for 0.20 time; from December 4 to December 8, 2013, Shanghai suffered the worst ever haze pollution, the air quality index (AQI) rose all the way up to 416, reaching Level 6 of serious pollution; and from 2014 to 2016 the over standard rate of ozone in Shenzhen was continuously high, and exceeded that of PM2.5 in 2015.
} 
actively summarize the urban wind environment and make space plan for typhoon prevention and treatment during site selection and construction of cities, we can greatly reduce losses, and save resources and reserves; at the same time, the change of urban spatial form would also affect air flow rate, smell diffusion velocity, wind speed comfort and other indexes of urban public space. Therefore, due to the rapid expansion of coastal cities in China, the development and change of urban spatial form has an important impact on the urban wind environment. Affected by typhoon, it has its particularity, complexity and contradiction. Strengthening the study on the coupling mechanism of coastal urban spatial form and wind environment will help to optimize the quality and process of urbanization in coastal areas of China. Wind environment affects cities' comfort level, air quality, building energy consumption and many other factors ${ }^{[2]}$. Wind sensitivity and wind load characteristics of buildings should be taken into consideration in the design and construction of urban buildings, roads and bridges. In the construction of urban square and park, the influence of wind environment also needs to be considered, so as to improve utilization rate and comfort of space. It can be seen that, it is necessary to optimize the urban spatial form in response to the demand for improving the quality of urban wind environment.

Wind environment is a complex interdisciplinary system, whose study can be divided into microscale, mesoscale and macroscale. Among them, microscale has little effect on the whole city, macroscale is too broad and not accurate enough; and mesoscale wind environment has important influence on the overall urban wind environment, and it is convenient to observe and collect data. For operability and depth of the study, we chose the mesoscale wind environment mainly representing the underlying surface of the city as the study object.

In addition, the multi-variable analysis method is good at dealing with complex variables. Using this feature and based on our previous accumulation, the research flexibly uses this analysis method to connect urban spatial form with mesoscale wind environment, forming a set of scientific and intact urban spatial form guidance system based on the optimization of urban mesoscale wind environment. It provides an objective and powerful scientific basis for the corresponding urban planning and design, and provides a reference for current hot spot study issues in China, such as haze governance, typhoon prevention and mitigation and energy saving and emission reduction.

Eventually, the study results can directly serve to the urbanization construction of coastal areas in China through the establishment of a multidimensional city information model in response to the concept of new smart city proposed by the central government in April 2016, and make contributions to the further acceleration of the implementation of city digitalization, network and intelligence. The study significance of this topic is mainly shown in the following three aspects:

- Significance of life: Coastal cities are featured by high density, high strength and great height. Roughness of city's underlying surface increases, so the near-surface wind environment becomes more complicated, which is not good for the safety and comfort of people's life and production. Wind environment has an influence on people's eating, wearing, living, using, traveling, entertainment and other aspects. The suitable wind environment is good for people's production and life, and will give people a more pleasant psychology. Therefore, it is necessary to summarize the coastal urban spatial form to adapt and optimize their urban wind environment.

- Ecological significance: If the urban mesoscale wind environment is optimized by improving the urban spatial form, the overall urban wind environment can be improved and energy consumption of buildings can be reduced. Good wind environment is reflected in good natural ventilation in summer and transition seasons and anti-wind measures in typhoon season, reduction of the use of air conditioning and heating facilities, the use of ecological and energy-saving method, prevention of intensifying of urban heat island effect and the occurrence of haze problem, etc., so that people and nature can coexist more harmoniously.

- Social significance: It is an urgent need for the healthy development of cities to provide the solution to optimization of spatial form in the urban planning and design, so as to deal with the wind environment problems which are easy to appear in cities. The construction of "smart city" will speed up the transformation and upgrading of industrial structure of cities, build a modern industrial system, promote the substantial development of the overall social economy, etc. 


\section{Research content}

Based on the study and screening of domestic typical cases of coastal cities of spatial form and characteristics of the wind environment, a feasible technology approach with which coastal urban spatial form and mesoscale wind environment system are connected with data and integrated is studied, and the mechanism of influence of change of coastal urban spatial form on wind environment is verified. Based on the energy balance mode in which the mesoscale wind environment is nested in the urban boundary layer and combined with the underlying surface of cities, the study makes the strategies of response by the urban spatial form to urban wind environment; simulates and analyzes response characteristics of urban heat island, local wind and pollution diffusion under different scenarios; combines with development demands of society, economy, resources and environment; and determines the feasible urban spatial form scheme. Finally, the study results are converted into a feasible scheme which can serve the society. The main study content can be divided into the following three aspects.

Study on the mechanism of influence of coastal urban spatial form on mesoscale wind environment. By comparing the coupling characteristics of spatial form and mesoscale wind environment of typical coastal cities in China, representative cities are selected as study cases. According to the characteristics of coastal cities, the method of quantitative evaluation of urban spatial form and micro climate in the underlying surface of cities is established; the basic rules of urban spatial form's coupling of the evolution of mesoscale wind environment are explored; the numerical models for wind and thermal environment of city buildings and streets are simulated; the parameter system of the coupling of the overall urban spatial form and wind environment is established; and calculation and analysis are made by flexibly using clustering analysis method, regression analysis method and other methods in the multi-variable analysis. The mechanism of influence of spatial form on mesoscale wind environment is summarized and verified by the wind tunnel test. From the global perspective, the mechanism of influence of spatial form on mesoscale wind environment in coastal cities in China is judged, and the international reference is provided for the improvement of urban wind environment in China.

Study on strategies of response by spatial form to mesoscale wind environment in coastal cities. In recent years, during the rapid expansion of coastal cities in Chin, their unreasonable planning and layout may cause deterioration of local climate conditions and ecological environment of cities, which have become one of the important factors restricting the sustainable development of cities. Complex terrain, mega cities, wind field effect of drainage basin, and the mechanism of interaction between the overall city and wind field in the terrain are studied. Through the simulation test in which the dynamic model of system is coupled with the scale model in the atmosphere, the mechanism of interaction between different forms and scales of spatial form and local microclimate effect and air quality is studied. Strategies of response by a series of coastal urban spatial form scenarios to the optimization of the mesoscale wind environment are summarized. The environmental factors involved include wind field, temperature field, humidity distribution, the distribution range of small wind areas, the ability of diffusion of atmospheric pollution, human comfort etc. The study contents are helpful to the optimization of urban planning, prevention and treatment of urban heat island, typhoon prevention and mitigation, energy saving and emission reduction, control of air pollution, etc.

Study on the spatial form guidance system coupled with mesoscale wind environment in coastal cities. The multi-variable analysis technology has been studied deeply by the applicant team in recent years after the introduction of Professor SAKAI Takeru. Through support vector machine and canonical variate analysis, this technology can accurately reflect the multi-variable autocorrelation model of the actual process, and construct various types of control systems for the multi-variable autocorrelation process. When events are observed from different angles, corresponding multi-variable results will be produced, and the variables are closely related to each other, but the data types may vary. Based on extraction and coupling of multi-angle variables of wind environment and urban spatial form, the topic tries to develop a multi-result variable system which can make statistics of, analyze and combine changes of data type, guide the expansion and 
rectification of urban spatial form, and improve the overall quality of wind environment in coastal cities.

\section{Research objectives}

Combined with the trend of acceleration of urbanization in the coastal areas year by year, and a series of wind environment problems brought by it, the topic studies a feasible technology approach for the coupling between multi-angle variables of coastal urban spatial form and the mesoscale wind environment system, so as to adapt to and further optimize the quality of wind environment in coastal cities, and achieve the target of disaster prevention and mitigation, energy saving and emission reduction. The topic further explores of service to the construction of smart city and digital city with the study results, tries to study and develop system software on the basis of the topic, so that it can provide services to more areas. At the same time, it can provide a reference for the global haze prevention and control, urban heat island circulation, global warming and other hottest researches.

\section{Key scientific problems to be solved}

The selection of cases, restrictive conditions and scope of coastal cities. Because of the great changes of coastline in China, the coastal urban forms and wind environment in different climatic zones vary a lot, and the nature and scale of cities are different. The study cases selected must be representative, and the comparability and validity of data shall be ensured. In the topic, we try to analyze from perspectives such as the global climate zone, urban scale and coastal features, simplify, establish a model, and make the secondary classification, so as to break through the difficulties.

The construction of the corresponding parameter system in which mesoscale wind environment and urban spatial form are effectively connected. The observation of events from multiple angles can produce corresponding multi-result variables, which are closely related to each other. But the data types may vary. How to build a multi-result variable system which can make statistics of, analyze and combine changes of data type, guide the expansion and rectification of urban spatial form and improve the overall quality of wind environment in coastal cities according to the actual situation is also a key scientific problem to be solved by the topic.

The construction of the mechanism of coupling between coastal urban spatial form and mesoscale wind environment. Based on the complexity, contradiction, uncertainty and versatility of study parameters, the corresponding variable system is established for the statistical analysis. After study on influence mechanism and response strategies of coastal urban spatial form on the mesoscale wind environment, the coupling mechanism between the two can be summarized one by one.

\section{Conclusions}

The feature and innovation of the research subject. There have been a lot of in-depth qualitative studies conducted on urban wind environment, local micro climate and other aspects in recent years, and various quantitative studies on urban spatial form, which lays a solid foundation for the study of the topic; and we will connect the two on this basis, actively do the quantitative study on the coupling mechanism between the two from the perspective of city, and further build an application software system. This project is characterized by the provision of basis for typhoon prevention and reduction, air pollution prevention and treatment, energy saving and emission reduction and other constructions of coastal cities in China.

The feature and innovation of the research method. Based on the complexity and contradiction of the wind environment of coastal cities and the complex and multi-variable characteristics of urban spatial form, the multi-variable analysis technology is the best choice for dealing with complex variables. In fact, due to its advantages in statistical analysis, it has been widely used in many industries, but it is still in the exploratory stage in the analysis and application of urban spatial 
form. Therefore, it is appropriate to systematically connect and analyze the two with the multi-variable analysis technology, and it is also the innovative application of a scientific study method.

The feature and innovation of the interdisciplinary integration. The topic is based on city planning, city design, architecture and other disciplines, and cooperates with material engineering, fluid mechanics, climate and meteorology, statistics and other fields. This is the inevitable trend in response to the development of the information age, and one of the characteristics of the project. In addition, the integration of resources from many universities is another feature.

\section{Acknowledgements}

This work was financially supported by the National Natural Science Foundation (51408118) and the State Bureau of Foreign Experts in High-end Projects (GDT20163600002) of China.

\section{References}

[1] An Initial Urban Wind Field Study in Washington, and DC (AugDavisurban). "An Initial Urban Wind Field Study in Washington, DC." Third Symposium on the Urban Environment.

[2] Razak, Azli Abd, et al. "Analysis of airflow over building arrays for assessment of urban wind environment." Building \& Environment 59.1(2013):56-65.

[3] Yuan, Chao, and E. Ng. "Building porosity for better urban ventilation in high-density cities - A computational parametric study." Building \& Environment 50.4(2012):176-189.

[4] $\mathrm{Ng}$, Edward, et al. "Improving the wind environment in high-density cities by understanding urban morphology and surface roughness: A study in Hong Kong." Landscape \& Urban Planning 101.1(2011):59-74.

[5] Sharples S, Bensalem R. Airflow in courtyard and atrium buildings in the urban environment: a wind tunnel study [J]. Solar Energy, 2001, 70(3):237-244.

[6] Schwerdtfeger, P., and T. J. Lyons. "Wind field studies in an urban environment." Urban Ecology 2.2(1976):93-107. 